\title{
Polarization-Basis Tracking Scheme in Satellite Quantum Key Distribution
}

\author{
Morio Toyoshima, ${ }^{1}$ Hideki Takenaka, ${ }^{1}$ Yozo Shoji, ${ }^{1}$ Yoshihisa Takayama, ${ }^{1}$ \\ Masahiro Takeoka, ${ }^{2}$ Mikio Fujiwara, ${ }^{2}$ and Masahide Sasaki ${ }^{2}$ \\ ${ }^{1}$ Space Communications Group, New Generation Wireless Communications Research Center, National Institute of Information and \\ Communications Technology (NICT), 4-2-1 Nukui-Kita, Koganei, Tokyo 184-8795, Japan \\ ${ }^{2}$ Quantum ICT Group, New Generation Network Communications Research Center, National Institute of Information and \\ Communications Technology (NICT), 4-2-1 Nukui-Kita, Koganei, Tokyo 184-8795, Japan
}

Correspondence should be addressed to Morio Toyoshima, morio@nict.go.jp

Received 8 January 2011; Revised 7 March 2011; Accepted 6 April 2011

Academic Editor: Ivan Djordjevic

Copyright ( $\odot 2011$ Morio Toyoshima et al. This is an open access article distributed under the Creative Commons Attribution License, which permits unrestricted use, distribution, and reproduction in any medium, provided the original work is properly cited.

Satellite quantum key distribution is a promising technique that overcomes the limited transmission distance in optical-fiber-based systems. The polarization tracking technique is one of the key techniques in the satellite quantum key distribution. With free-space quantum key distribution between an optical ground station and a satellite, the photon polarization state will be changed according to the satellite movement. To enable polarization based quantum key distribution between mobile terminals, we developed a polarization-basis tracking scheme allowing a common frame of reference to be shared. It is possible to orient two platforms along a common axis by detecting the reference optical signal only on the receiver side with no prior information about the transmitter's orientation. We developed a prototype system for free-space quantum key distribution with the polarization-basis tracking scheme. Polarization tracking performance was $0.092^{\circ}$ by conducting quantum key distribution experiments over a $1 \mathrm{~km}$ free space between two buildings in a Tokyo suburb.

\section{Introduction}

Quantum key distribution (QKD) was first experimentally demonstrated with polarization photons in 1984 by Bennett and Brassard, who achieved over a free-space distance of $30 \mathrm{~cm}[1,2]$. Shortly, QKD experiments with polarization coding in optical fibers were performed [3]. Due to the random nature of polarization fluctuations, active polarization tracking devices [4] or polarization diversity schemes [5] must be used. The transmission distance in optical fibers has been extended with active polarization control schemes for quantum communication [6, 7]; however, the transmission distance is limited to around $140 \mathrm{~km}$ because of optical loss and background noise in the fibers. In both cases, the QKD session was interrupted at times to perform the polarization tracking. The compensation was restricted only to polarization variations slow enough to allow key transmission without control for a while. The quantum communication experiment was performed with the real-time continuous polarization control in optical fibers [8].

On the other hand, free-space QKD between an optical ground station (OGS) and a satellite can extend the transmission distance in quantum networks beyond that achieved with optical fibers, and the polarization is stable in free-space transmission, which is considered to be freespace's key advantage and an important application. Freespace QKD experiments have been conducted and the freespace distance was extended to about $140 \mathrm{~km}$ [9-13]. The feasibility of using quantum cryptography for secure satellite communications was investigated by Hughes et al. in 2000 [14]. Recently, laser communication experiments between satellites and OGSs were successfully demonstrated [15-17], and similar laser communication terminals are used in the satellite QKD [18-20]. However, one of the key techniques in the satellite $\mathrm{QKD}$ is the polarization tracking technique because the photon polarization state will be changed 


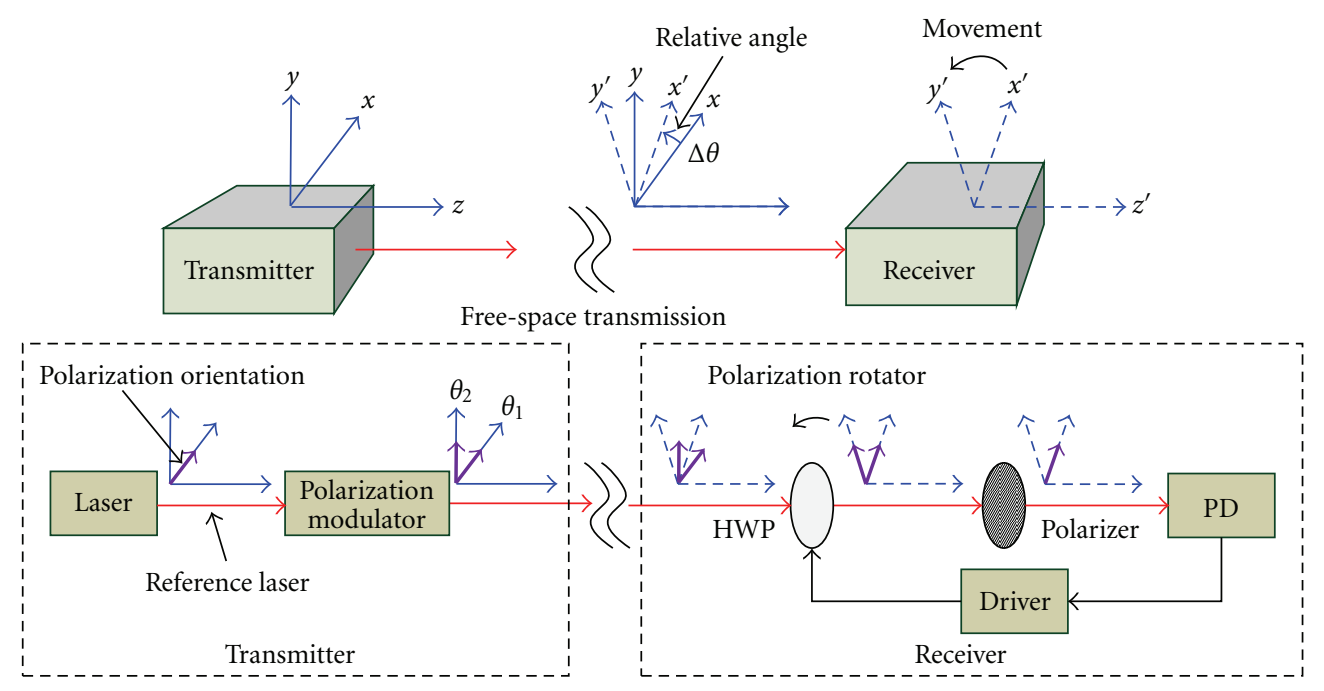

FIgURE 1: Configuration of the polarization-basis tracking scheme at the transmitter.

according to the satellite movement. To enable polarizationbased QKD between mobile terminals, we developed a novel polarization-basis tracking scheme allowing a common frame of reference to be shared. It is possible to achieve an alignment of two polarization orientations on the different platforms along a common axis by detecting the reference laser polarization at the receiver side. The depolarization effect between an LEO satellite and an OGS will be small [21] but the dynamic satellite movement must be compensated by employing the polarization tracking technique.

A key challenge in linking ground-to-satellite-based polarization QKD terminals is ensuring they share a common reference frame, that is, they are oriented such that "horizontal" maps to "horizontal" polarization, and so forth. Thus, for this purpose, the polarization-basis tracking scheme that can detect the rotational orientation at the receiver side with no prior information about that at the transmitter side is proposed. Section 2 describes the principle of the proposed polarization tracking scheme. Section 3 shows the configuration of a prototype system developed for free-space QKD with the polarization-basis tracking scheme. The polarization tracking performance of the system was investigated in QKD experiments performed over a free space of $1 \mathrm{~km}$ between two buildings in suburban Tokyo, with the obtained results described in Section 4. By using the measured parameters, the performance of decoy-state QKD was evaluated to study the feasibility of enabling longer distances and faster key rates between the earth and a satellite.

\section{Principle of Polarization Tracking Scheme}

In the satellite $\mathrm{QKD}$, the polarization variation due to the atmospheric effect between the satellite and the OGS is small [21]; however; the photon polarization orientation is dynamically changed because of the relative movement due to the satellite orbit. Therefore, the polarization tracking technique is one of the key techniques in the satellite QKD. Figure 1 shows the configuration of the polarization tracking scheme, and the definition of each axis between the transmitter in $(x, y, z)$ coordinates and receiver in $\left(x^{\prime}, y^{\prime}, z^{\prime}\right)$ coordinates is shown. The transmitter consists of the reference laser at the $1.5 \mu \mathrm{m}$ wavelength and the polarization modulator. The polarization orientation of the reference laser is adjusted and modulated so it is in the same direction as one of the polarization bases of the $0.8 \mu \mathrm{m}$ weak coherent pulse (WCP) for the quantum optical signal. The receiver consists of the polarization rotator with a half-wave plate (HWP), polarizer, and photodiode (PD). The polarization rotator includes a stepping motor, a control system, and the HWP for both wavelengths of $0.8 \mu \mathrm{m}$ and $1.5 \mu \mathrm{m}$. The alignment of each terminal can be first compensated by a tip-tilt tracking system, and then the terminal's polarization orientation is adjusted along a common axis by using the proposed polarization tracking system.

The modulation waveforms for different polarization bases of the reference laser are shown in Figure 2 as a function of the angular difference of $\Delta \theta$. The angular difference of $\Delta \theta$ is defined as the angle between the transmitter and receiver orientations as shown in Figure 1. The polarization orientation at $1.5 \mu \mathrm{m}$ is modulated with two polarizations of $\theta_{1}$ and $\theta_{2}$ in Figure 2(a) with a frequency which is independent from the WCP. In this case, $\theta_{1}$ and $\theta_{2}$ are set to $0^{\circ}$ and $90^{\circ}$, respectively. The optical signals after the polarizer received by the PD are shown in Figures 2(b)-2(e) as a function of the angular difference of $\Delta \theta$. The clock recovery function in the receiver can synchronize the transmitted modulated waveform. The waveform of the modulated polarization signal in Figure 2(a) is in the same phase as that of the output signal in Figure 2(d) when the angular difference is $45^{\circ}<|\Delta \theta|<135^{\circ}$. The waveforms of the output optical signals (Figures 2(b) and 2(c)) are inverted, with an angular difference of $0^{\circ} \leq|\Delta \theta|<45^{\circ}$. The phase relation between the transmitter and receiver when the angular difference is $135^{\circ}<|\Delta \theta|<180^{\circ}$ becomes identical to that when the angular difference is $0^{\circ}<|\Delta \theta|<45^{\circ}$. Thus, the polarization 


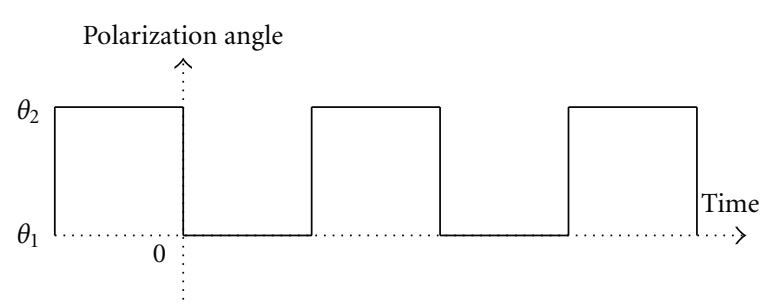

(a)

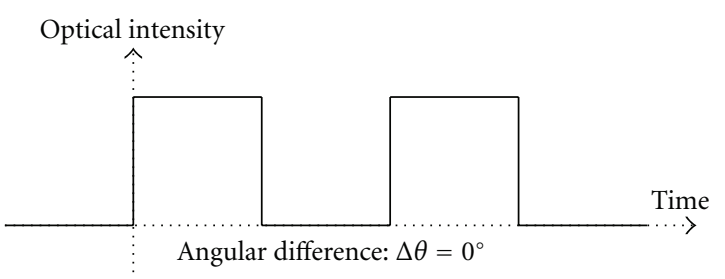

(b)

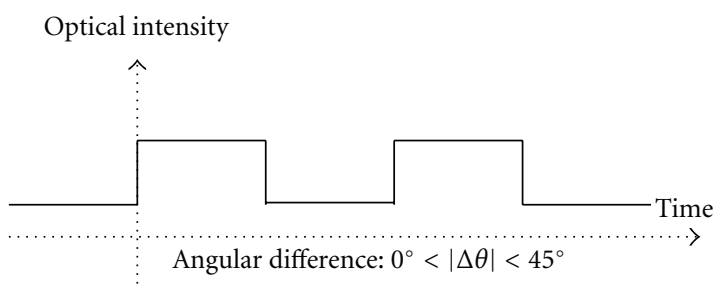

(c)

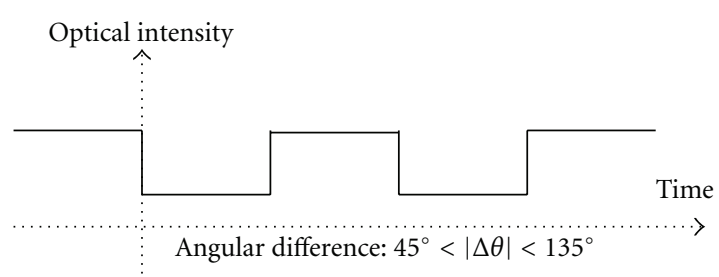

(d)

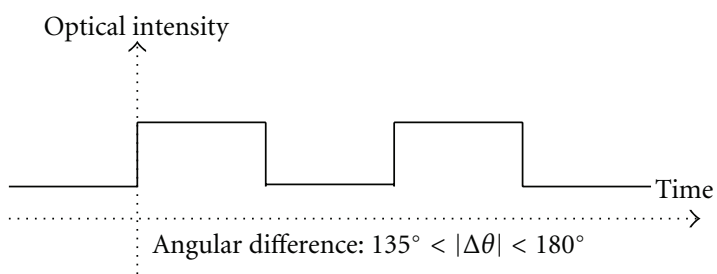

(e)

FIGURE 2: Modulation waveforms for different polarization bases of the reference beam: (a) polarization modulation, (b)-(e) received signals as a function of the relative angular difference between the transmitter and receiver orientations.

orientation of the transmitter can be detected on the receiver side by the amplitude and phase information on the received signal waveform of the reference $1.5-\mu \mathrm{m}$ laser beam.

The output signals measured by a $1.5-\mu \mathrm{m}$ PD after the polarizer are shown in Figures 3(a), 4(a), and 5(a) for $0^{\circ}<|\Delta \theta|<45^{\circ}, \Delta \theta=45^{\circ}$, and $45^{\circ}<|\Delta \theta|<$ $135^{\circ}$, respectively. The waveforms of the applied voltage for the polarization rotator are shown in Figures 3(b), 4(b),

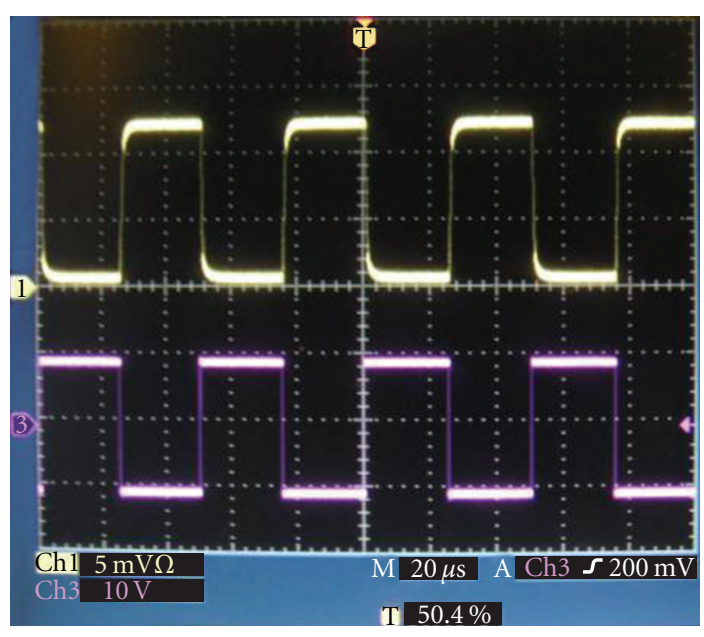

Figure 3: $1.5-\mu \mathrm{m}$ optical output signal after polarizer with the angular difference between the transmitter and receiver of $0^{\circ} \leq$ $|\Delta \theta|<45^{\circ}$. (a) The $1.5-\mu \mathrm{m}$ optical output signal after the polarizer and (b) the applied voltage $(20: 1)$ for the polarization modulator are shown as Ch1 and Ch3 with the modulated frequency of $20 \mathrm{kHz}$.

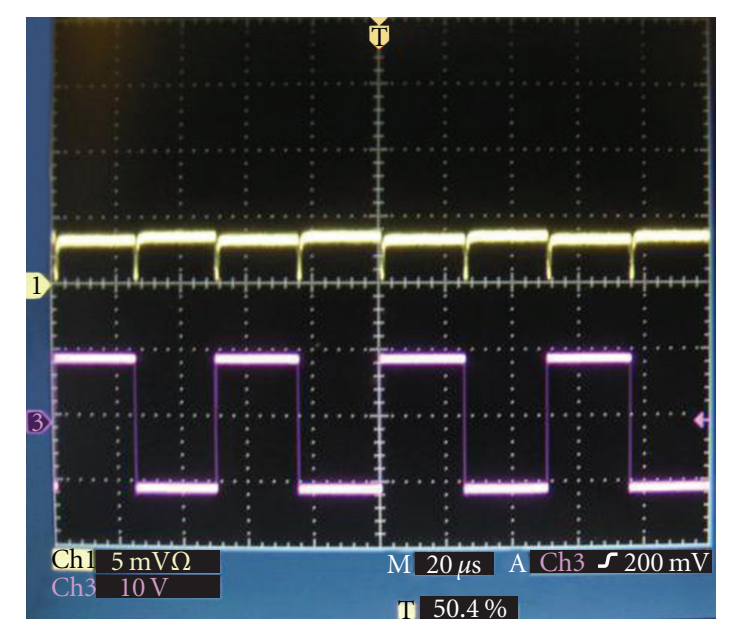

FIGURE 4: $1.5-\mu \mathrm{m}$ optical output signal after polarizer with the angular difference between transmitter and receiver of $|\Delta \theta|=45^{\circ}$. (a) The $1.5-\mu \mathrm{m}$ optical output signal after the polarizer and (b) the applied voltage $(20: 1)$ for the polarization modulator are shown as $\mathrm{Ch} 1$ and $\mathrm{Ch} 3$ with the modulated frequency of $20 \mathrm{kHz}$.

and 5(b), respectively. The modulated frequency is $20 \mathrm{kHz}$. Figure 4(a) has the same phase waveform as that of the applied voltage in Figure 5(b) when the angular difference is $45^{\circ}<|\Delta \theta|<135^{\circ}$. The waveforms of the output optical signal and the applied voltage signal in Figures 4(a) and 4(b) are inverted with an angular difference of $0^{\circ} \leq|\Delta \theta|<$ $45^{\circ}$. The amplitude of the optical output signal becomes a minimum when the angular difference is $|\Delta \theta|=45^{\circ}$ as shown in Figure 4(a). Thus, the polarization orientation of the transmitter can be detected only from the optical output signal of the $1.5-\mu \mathrm{m}$ laser beam after the polarizer, and the control loop of the polarization rotator operates so that the amplitude of the output signal can be the minimum. 


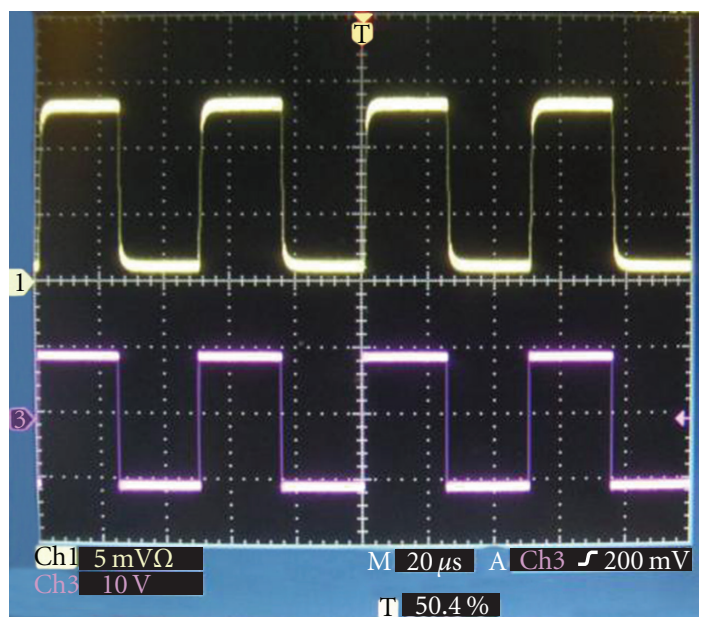

FIGURE 5: $1.5-\mu \mathrm{m}$ optical output signal after polarizer with the angular difference between transmitter and receiver of $45^{\circ}<|\Delta \theta|<$ $135^{\circ}$. (a) The $1.5-\mu \mathrm{m}$ optical output signal after the polarizer and (b) the applied voltage $(20: 1)$ for the polarization modulator are shown as Ch1 and Ch3 with the modulated frequency of $20 \mathrm{kHz}$.

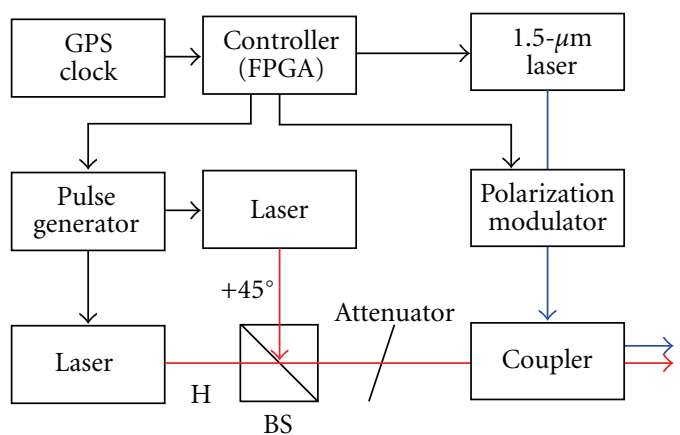

Figure 6: Configuration of transmitter (Alice).

The tracked orientation at the minimum amplitude can be chosen freely by the orientation of the polarizer according to the internal optical axis in the receiver.

\section{Prototype Model for Free-Space QKD}

3.1. System Configuration. A prototype free-space QKD system with the WCP and polarization-basis tracking scheme was developed. The prototype model operates at a clock rate of $100 \mathrm{MHz}$. The system uses the B92 protocol [22] for QKD owing to the simplicity of the transmitter system. This is because, unlike the BB84 protocol that uses four laser diodes, the $\mathrm{B} 92$ protocol requires only two laser diodes and only one polarization basis. The WCP signals modulated at two polarization states and randomly transmitted. One of the photon polarization states is aligned along the one of the polarization orientation of the reference laser beam. About the security issue, the information on the polarization state can be eavesdropped but the information on the quantum bits itself cannot be eavesdropped. The configuration of the transmitter (Alice) is shown in Figure 6. The wavelength of

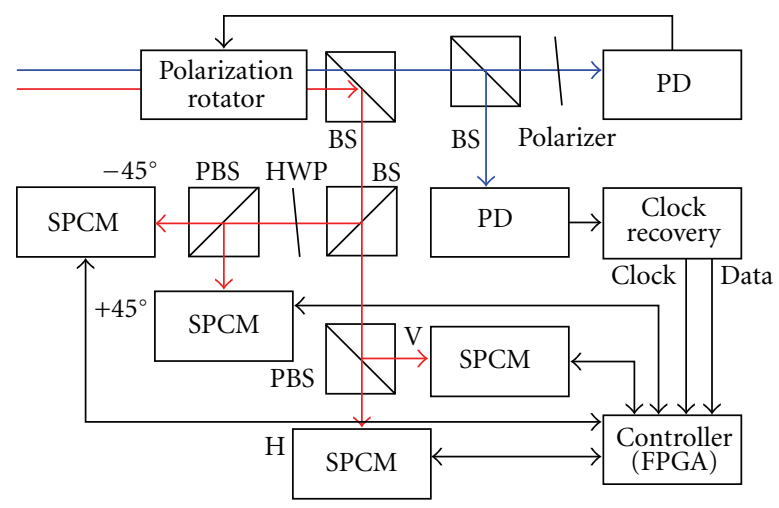

Figure 7: Configuration of receiver (Bob).

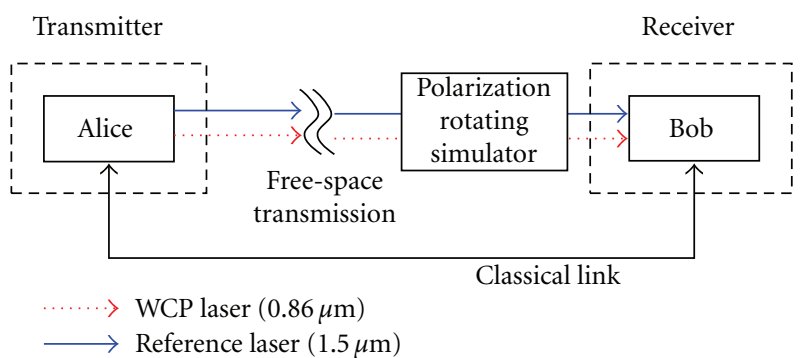

Figure 8: Configuration of the experiment.

the lasers for the single-photon quantum channel is $0.86 \mu \mathrm{m}$. The secret bits are sent as the WCP signals at $0.86 \mu \mathrm{m}$. The two laser diodes for polarization at $0^{\circ}$ and $+45^{\circ}$ are selected on the basis of randomly generated data. A 1.5$\mu \mathrm{m}$-wavelength, laser is used for the polarization orientation tracking and the synchronization of the $0.86-\mu \mathrm{m}$ WCP signals. The transmitter has three functions: synchronization of the GPS clock, generation of communication signals, and generation of random signals for polarization modulation. The signal processing aspect consists of a fieldprogrammable gate array (FPGA), which records all data in the data storage computer in the transmitter. The configuration of the receiver (Bob) is shown in Figure 7. In the receiver, the GPS, clock data recovery, and quantum signals detected by single-photon counting modules (SPCMs) and random signals for polarization modulation are synchronized. All data are saved electronically in the receiver. The receiver contains optical filters for reducing the background level.

3.2. Characteristics of Polarization-Basis Tracking. The rotation of the terminal's orientation was simulated by the polarization rotating simulator which consists of an HWP rotator for both wavelengths of $0.8 \mu \mathrm{m}$ and $1.5 \mu \mathrm{m}$, and placed in front of the receiver, as shown in Figure 8. The reference laser beam at $1.5-\mu \mathrm{m}$ was transmitted to the receiver. The intended rotation angle can be added to the incident polarization bases by the polarization rotating simulator in front of the receiver. The rotated angle of the polarization was compensated by using the proposed polarization tracking scheme in the receiver. Figure 9 shows the compensated motor rotation 


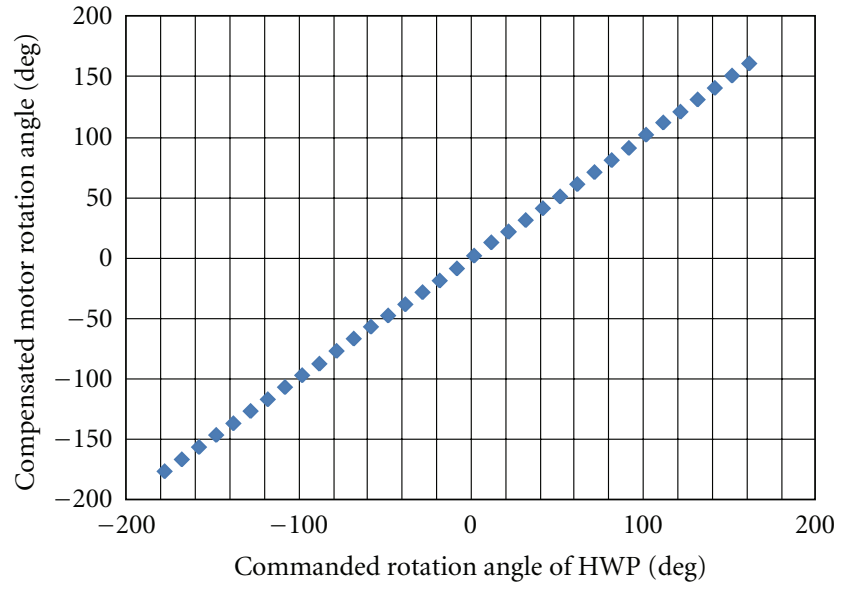

FIgURE 9: Compensated motor rotation angle of the polarization tracking system in the receiver as a function of commanded rotation angle of the half-wave plate in the polarization rotating simulator in front of the receiver.

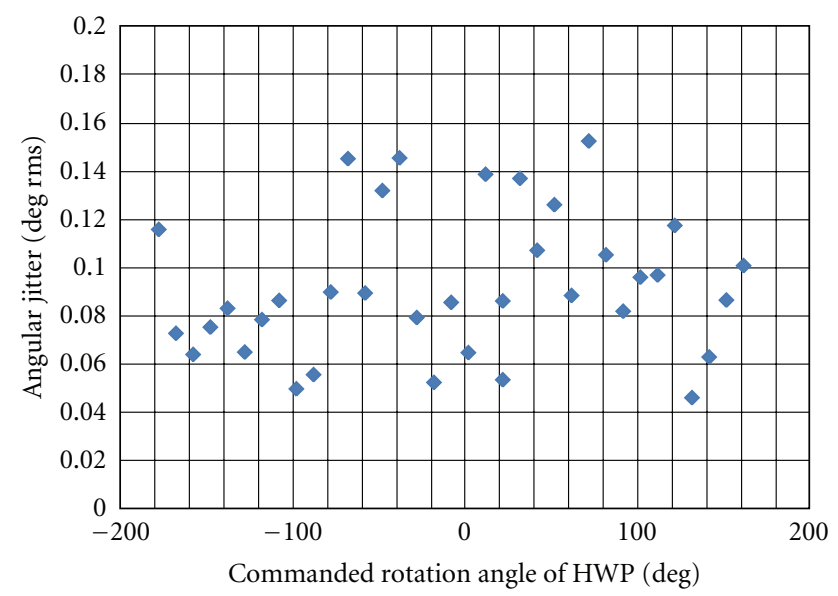

FIGURE 10: Rms angular jitter error of the polarization tracking system in the receiver as a function of commanded rotation angle of the half-wave plate in the polarization rotating simulator in front of the receiver.

angle in the receiver against the commanded rotation angle of the HWP rotator in the polarization rotating simulator in front of the receiver measured in the laboratory. The polarization rotation characteristics were linear, and the rms (root mean square) angular jitter error in the polarization basis tracking system can be defined as

$$
\theta_{\text {rms }}=\sqrt{\frac{1}{N} \sum\left(\theta_{\text {measured }}-\theta_{\text {command }}\right)^{2}},
$$

where $\theta_{\text {command }}$ is the commanded angle in the polarization rotating simulator, $\theta_{\text {measured }}$ is the compensated angle in the receiver, and $N$ is the number of samples. The angular jitter error of the polarization tracking system in the receiver was estimated for each commanded angle in the polarization rotating simulator as shown in Figure 10. The rms angular jitter error was estimated to be $0.092^{\circ}$. This result showed that the tracking error of the proposed polarization-basis

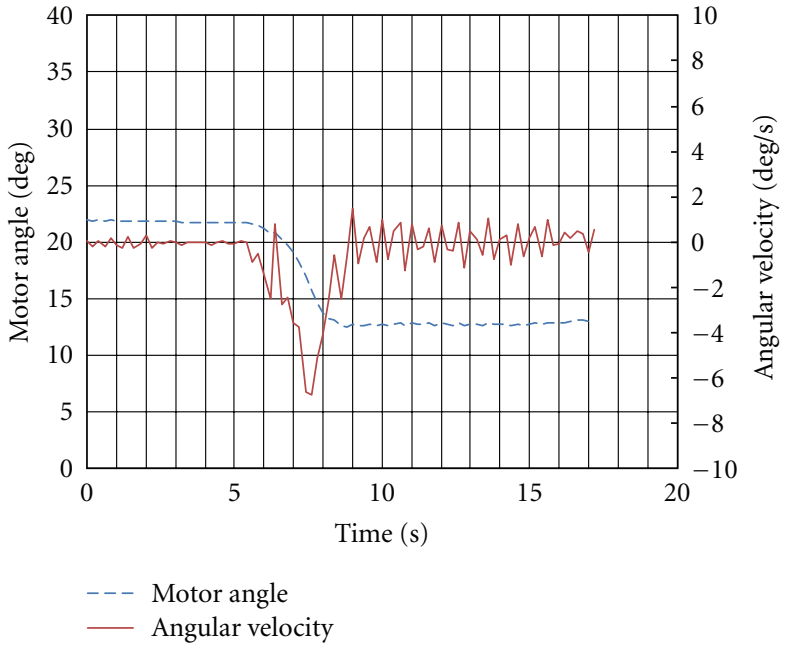

FIGURE 11: Rotation angle and angular velocity response of the motor when the step response with a 10-degree rotational angle is applied.

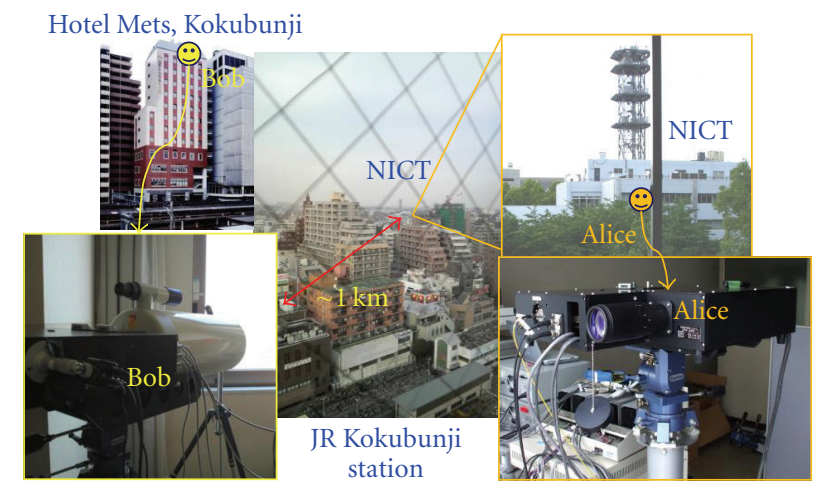

Figure 12: Photographs of field QKD experiments between two buildings in suburban Tokyo.

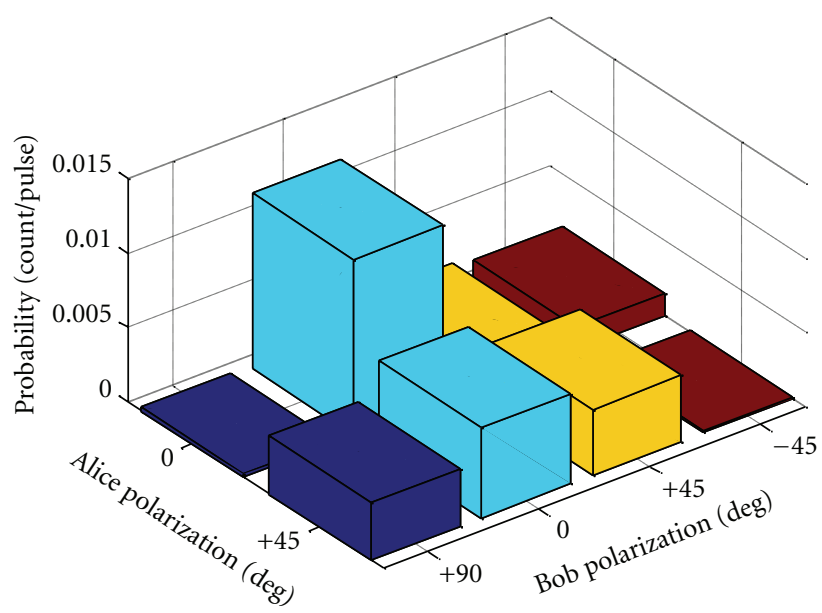

FIGURE 13: Relation between the preparation basis and measurement outcomes measured by Alice and Bob for randomly selected polarizations without the error correction. 
tracking scheme is small enough for application to satellite QKD systems. Figure 11 shows an example of the step response of the rotation angle and angular velocity of the stepping motor when a step angle of $10^{\circ}$ was applied to the HWP rotator in the polarization rotating simulator placed in front of the receiver in the laboratory. In this system, the angular velocity was approximately $7^{\circ} / \mathrm{s}$, which was 10 times larger than that required for the QKD terminals onboard the LEO satellites. The tracking loop converges within several seconds.

\section{Experimental Results for $1 \mathrm{~km}$ Free-Space QKD}

4.1. Polarization-Basis Tracking Error. Free-space QKD tests were performed at the NICT facilities one night in March 2009 over a distance of $1 \mathrm{~km}$ between the Hotel Mets at Tokyo's Kokubunji train station and the NICT building. Photographs of the equipment and test buildings are shown in Figure 12. The optical signals were sometimes interrupted by atmospheric turbulence, which caused signal fading in the $1.5-\mu \mathrm{m}$ classical communication link. In the field test conducted between the two buildings, the rms angular jitter error in the polarization-basis tracking system was measured to be $0.089^{\circ}$ as a constant rotation angle during tracking, which was close to the angular jitter error measured in the laboratory as shown in Figure 10. The obtained results showed that our polarization-basis tracking scheme worked well under the atmospheric turbulence condition in the $1 \mathrm{~km}$ laser link.

4.2. QKD Experiments. The relation between the preparation basis and measurement outcomes measured by Alice and Bob for randomly selected polarizations without the error correction is shown in Figure 13. The mean photon number $(\mu)$ of the receiver was set at 0.0962 photons/pulse during the preparatory test conducted in the laboratory. The relation of the preparation basis and measurement outcomes between Alice and Bob with the error correction is shown in Figure 14 for the sifted key data. The quantum bit error rate (QBER) was $0.57 \%$. The optical beam was defocused with a beam divergence angle of $0.6 \mathrm{mrad}$ to mitigate the tracking error in the transmitted laser beam. The loss of channel was $-10.3 \mathrm{~dB}$ in this configuration. Error bits were corrected by using the BBBSS protocol [2]. The sifted key rate was $240.21 \mathrm{kbps}$, as shown in Table 1.

4.3. Improvements for Decoy-State QKD System. For future improvement, the QKD with the decoy state can increase the further transmission distance [23]. The final key rates in the decoy state achieved with the BB84 protocol and the clock rate of $2.5 \mathrm{GHz}$ for the free-space and fiber-based transmission systems were estimated based on the parameter values measured in the 1-km field experiments (Figure 15), which can reflect the real field test environment for the QBER, background level, and so on. From the figure, it is apparent that the final key rates are improved with an improvement in the dark count rate of the optical systems.

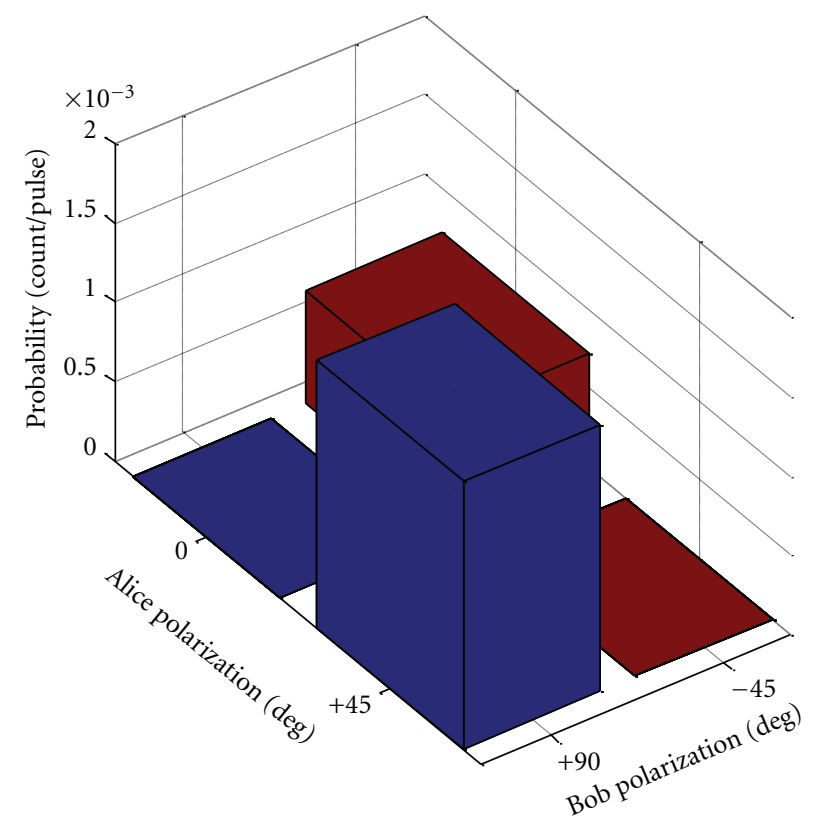

FIGURE 14: Relation between the preparation basis and measurement outcomes measured by Alice and Bob for randomly selected polarizations with the error correction.

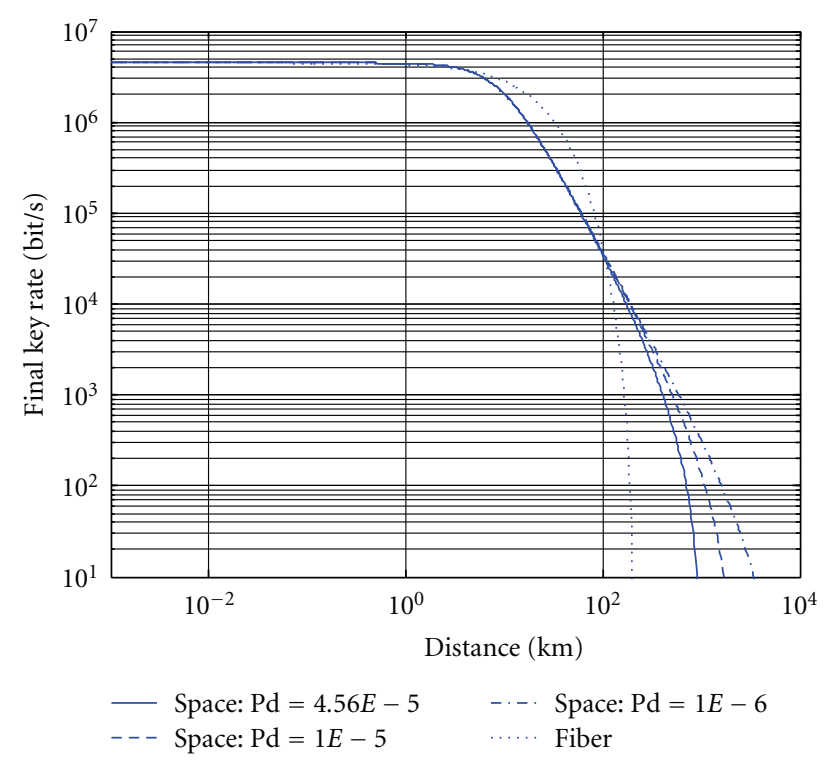

Figure 15: Final key rates achieved with BB84 and for the decoy state with $\mu=0.5$ photons/pulse for free-space and fiber-based transmission systems at the clock rate of $2.5 \mathrm{GHz}$ as a function of transmission distance. Pd stands for dark count rate in counts/ pulse. The rest of parameters corresponding to the prototype model in Table 1 are assumed.

The parameters for the prototype model are set using the data provided in Table 1 . The $\mu$ value in the decoy state is assumed to be 0.5 photons/pulse. As can be seen from Figure 15, an improvement in the dark count rate leads to an increase in the transmission distance. At the final 
TABLE 1: Parameters used in the 1-km QKD experiments.

\begin{tabular}{lc}
\hline Item & Value \\
\hline Clock rate & $100 \mathrm{MHz}$ \\
Protocol & $\mathrm{B} 92$ \\
Mean photon number & 0.0962 photons/pulse \\
Beam divergence angle & $0.6 \mathrm{mrad}$ \\
Dark count rate & $4.56 E-5$ counts/pulse \\
QBER & $0.57 \%$ \\
Sift key rate & $240.21 \mathrm{kbps}$ \\
Distance & $1.065 \mathrm{~km}$ \\
\hline
\end{tabular}

key rate, the maximum transmission distance in the freespace transmission system tends to be longer than that in the fiber-based system when the optical loss is $0.2 \mathrm{~dB} / \mathrm{km}$. By reducing the imperfections in the optical system, both the transmission distance and the final key rate can be increased. Though the final key rate is several tenths of a bit per second, QKD exchange will be possible, but a suitable system must be designed and developed for enabling QKD between the earth and a satellite. A transmission distance of several hundred kilometers will be required for LEO satellites. This distance can be increased to up to several thousand kilometers when the dark count rate is less than $1 E-6$ counts/pulse, and the final key rate can be increased to up to a few kilobits per second. For further improvement of the final key rate, the clock rate for QKD using satellites must be increased, and very fast response single-photon detectors, such as Superconducting Single-Photon Detectors (SSPDs), must be used in the future [24].

\section{Conclusion}

This paper proposed the polarization-basis tracking scheme and discussed the development of a free-space QKD system. The experimental results were shown in the field test carried out with a $1 \mathrm{~km}$ free-space laser link. A polarization tracking scheme that uses a polarization rotator was proposed, and the rms angular jitter error in the polarization-basis tracking system of $0.089^{\circ}$ was measured. By using a polarizationmodulated signal at a wavelength of $1.5 \mu \mathrm{m}$, the polarization could be determined when two mobile terminals were moving relative to one another. In this method, no prior information about the information of the satellite orientation was required. Therefore, the polarization tracking was necessary only on the receiver side. In the field QKD experiment, the sifted key rate was $240 \mathrm{kbps}$. The dark count rate for the photon-counting detectors was $4.56 E-5$ counts/pulse at night in a Tokyo suburb. The optical bandpass filter placed in front of the photon-counting detectors helped in reducing the dark count rate. Improvements made to the clock rate and dark count rate in the decoy state are expected to increase the transmissible distance to up to several thousand kilometers and the final key rate to up to a few kilobits per second. Thus the proposed scheme helps realize free-space QKD from LEO satellites.

\section{References}

[1] C. H. Bennett and G. Brassard, "Quantum cryptography: public key distribution and coin tossing," in Proceedings of the IEEE International Conference on Computers, Systems \& Signal Processing, pp. 175-179, Bangalore, India, 1984.

[2] C. H. Bennett, F. Bessette, G. Brassard, L. Salvail, and J. Smolin, "Experimental quantum cryptography," Journal of Cryptology, vol. 5, no. 1, pp. 3-28, 1992.

[3] A. Muller, J. Breguet, and N. Gisin, "Experimental demonstration of quantum cryptography using polarized photons in optical fibre over more than 1 km," Europhysics Letters, vol. 23, pp. 383-388, 1993.

[4] N. G. Walker and G. R. Walker, "Polarization control for coherent communications," Journal of Lightwave Technology, vol. 8, no. 3, pp. 438-458, 1990.

[5] L. G. Kazovsky, "Phase- and polarization-diversity coherent optical techniques," Journal of Lightwave Technology, vol. 7, no. 2, pp. 279-292, 1989.

[6] C. Z. Peng, J. Zhang, D. Yang et al., "Experimental longdistance decoy-state quantum key distribution based on polarization encoding," Physical Review Letters, vol. 98, no. 1, Article ID 010505, 4 pages, 2007.

[7] J. Chen, G. Wu, Y. Li, E. Wu, and H. Zeng, "Active polarization stabilization in optical fibers suitable for quantum key distribution," Optics Express, vol. 15, no. 26, pp. 17928-17936, 2007.

[8] G. B. Xavier, N. Walenta, G. V. De Faria et al., "Experimental polarization encoded quantum key distribution over optical fibres with real-time continuous birefringence compensation," New Journal of Physics, vol. 11, Article ID 045015, 2009.

[9] R. J. Hughes, J. E. Nordholt, D. Derkacs, and C. G. Peterson, "Practical free-space quantum key distribution over $10 \mathrm{~km}$ in daylight and at night," New Journal of Physics, vol. 4, pp. 1-14, 2002.

[10] C. Kurtsiefer, P. Zarda, M. Halder et al., "Quantum cryptography: A step towards global key distribution," Nature, vol. 419, no. 6906, p. 450, 2002.

[11] T. Schmitt-Manderbach, H. Weier, M. Fürst et al., "Experimental demonstration of free-space decoy-state quantum key distribution over 144 km," Physical Review Letters, vol. 98, no. 1, Article ID 010504, 4 pages, 2007.

[12] C.-Z. Peng, T. Yang, X.-H. Bao et al., "Experimental free-space distribution of entangled photon pairs over $13 \mathrm{~km}$ : Towards satellite-based global quantum communication," Physical Review Letters, vol. 94, no. 15, Article ID 150501, 4 pages, 2005.

[13] R. Ursin, F. Tiefenbacher, T. Schmitt-Manderbach et al., "Freespace distribution of entanglement and single photons over 144 km," Quantum Physics, vol. 3, pp. 481-486, 2007.

[14] R. J. Hughes, W. T. Buttler, P. G. Kwiat et al., "Quantum cryptography for secure satellite communications," in Proceedings of the IEEE Aerospace Conference, pp. 191-200, March 2000.

[15] K. E. Wilson, J. R. Lesh, K. Araki, and Y. Arimoto, "Overview of the ground-to-orbit lasercom demonstration," Space Communications, vol. 15, no. 2, pp. 89-95, 1998.

[16] T. T. Nielsen and G. Oppenhaeuser, "In orbit test result of an operational optical intersatellite link between ARTEMIS and SPOT4, SILEX," in Proceedings of The 3rd International Society for Optical Engineering (IAC '02), pp. 1-11, Houston, Tex, USA, 2002.

[17] M. Toyoshima, K. Takizawa, T. Kuri et al., "Development of the optical ground station for the oicets satellite and experimental results," in Proceedings of the 57th International Astronautical Congress (IAC '06), pp. 1-11, Valencia, Spain, October 2006. 
[18] M. Aspelmeyer, T. Jennewein, M. Pfennigbauer, W. R. Leeb, and A. Zeilinger, "Long-distance quantum communication with entangled photons using satellites," IEEE Journal on Selected Topics in Quantum Electronics, vol. 9, no. 6, pp. 15411551, 2003.

[19] R. Kaltenbaek, M. Aspelmeyer, T. Jennewein et al., "Proofof-concept experiments for quantum physics in space," in The International Society for Optical Engineering, vol. 5161 of Proceedings of SPIE, pp. 252-268, 2004.

[20] M. Pfennigbauer, M. Aspelmeyer, W. R. Leeb et al., "Satellitebased quantum communication terminal employing state-ofthe-art technology," Journal of Optical Networking, vol. 4, no. 9, pp. 549-560, 2005.

[21] M. Toyoshima, H. Takenaka, Y. Shoji, Y. Takayama, Y. Koyama, and H. Kunimori, "Polarization measurements through spacetoground atmospheric propagation paths by using a highly polarized laser source in space," Optics Express, vol. 17, no. 25, pp. 22333-22340, 2009.

[22] C. H. Bennett, "Quantum cryptography using any two nonorthogonal states," Physical Review Letters, vol. 68, no. 21, pp. 3121-3124, 1992.

[23] H. K. Lo, X. Ma, and K. Chen, "Decoy state quantum key distribution," Physical Review Letters, vol. 94, no. 23, Article ID 230504, pp. 1-4, 2005.

[24] S. Miki, M. Fujiwara, M. Sasaki, and Z. Wang, "NbN superconducting single-photon detectors prepared on single-crystal $\mathrm{MgO}$ substrates," IEEE Transactions on Applied Superconductivity, vol. 17, no. 2, pp. 285-288, 2007. 

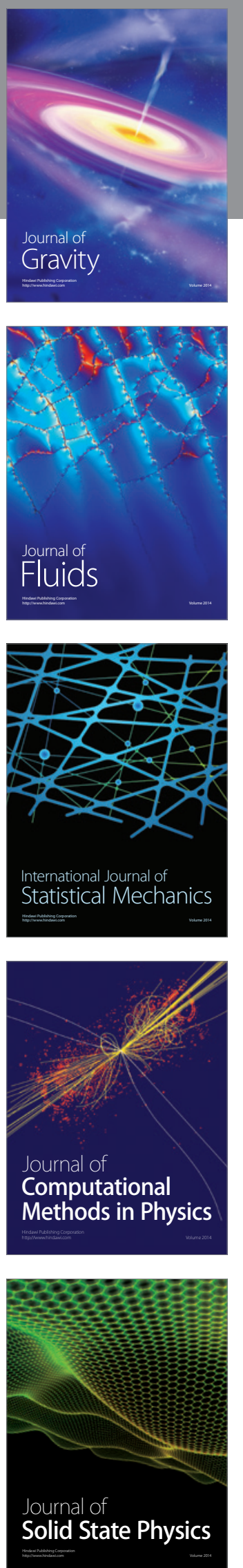

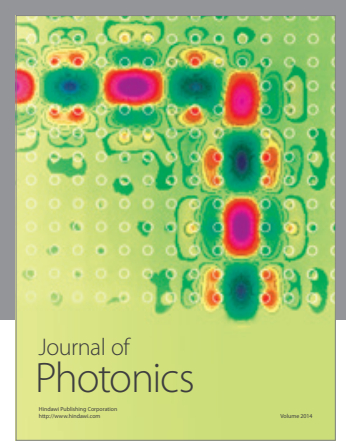

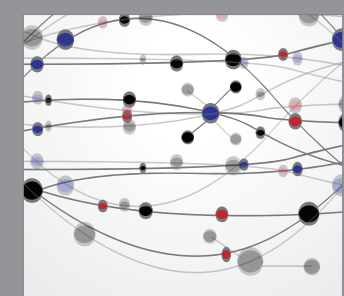

The Scientific World Journal
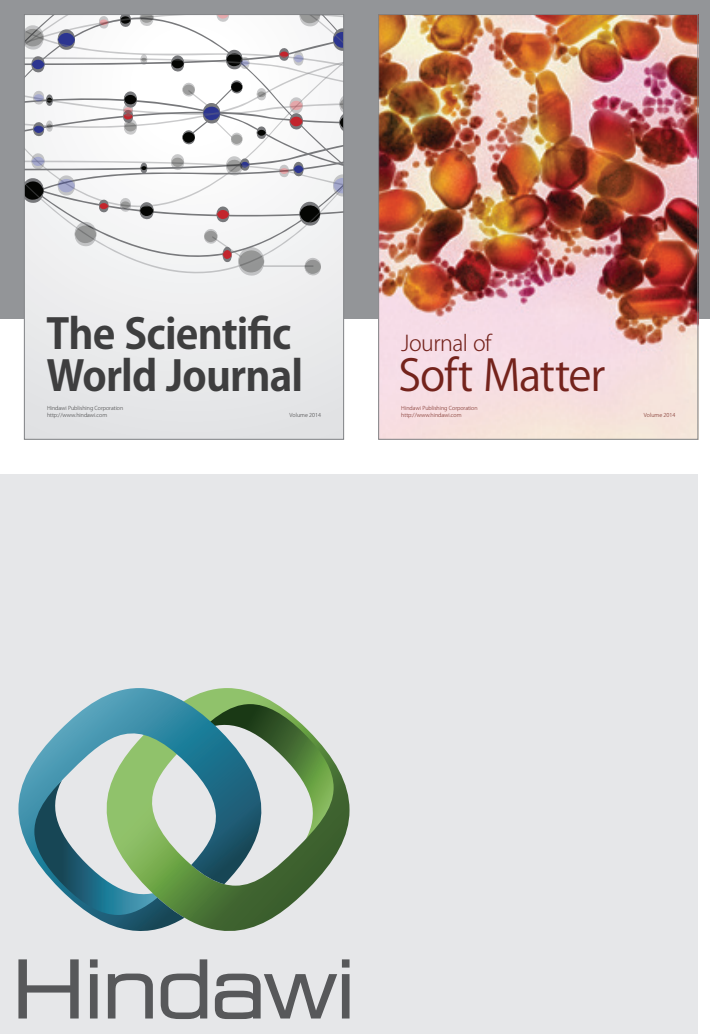

Submit your manuscripts at

http://www.hindawi.com
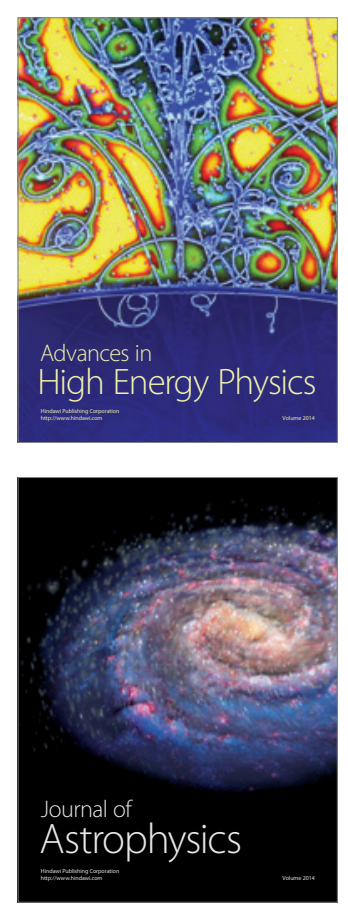
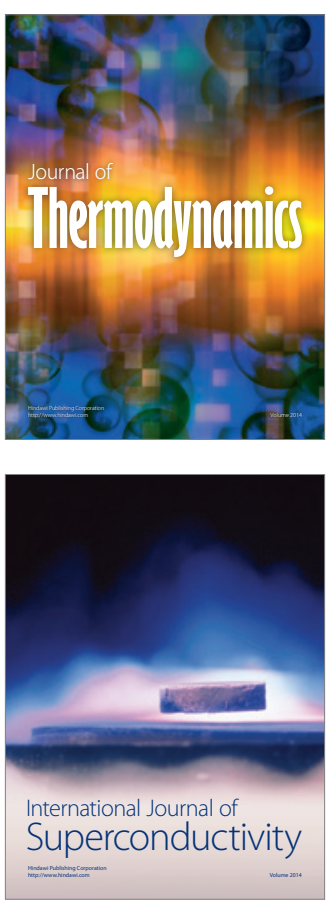
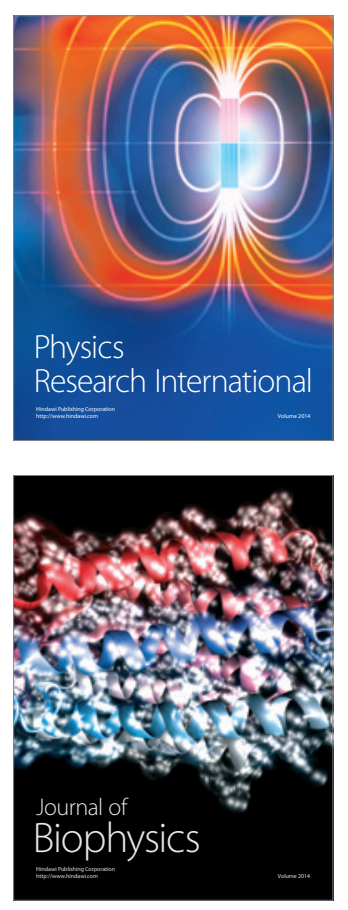
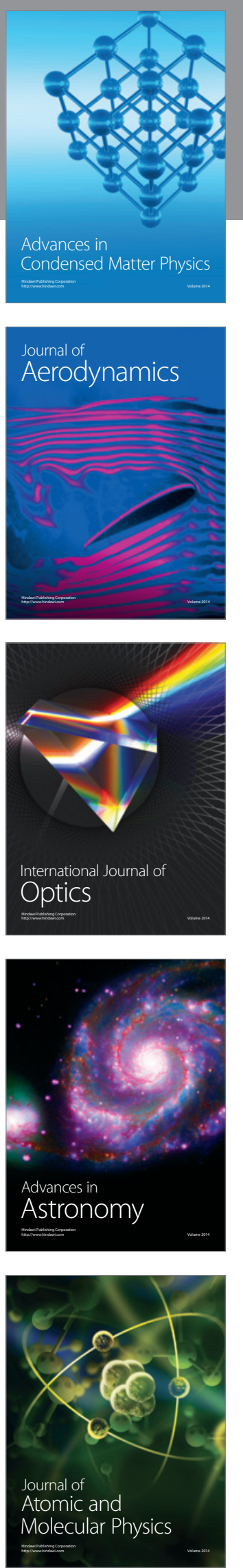